\title{
Rovibrational structure of the Ar-CO complex based on a novel three-dimensional $a b$ initio potential
}

\author{
Thomas Bondo Pedersen a) \\ Department of Physical Chemistry, Institute of Molecular Science, University of Valencia, \\ E-46100 Burjassot, Valencia, Spain \\ Javier López Cacheiro and Berta Fernández \\ Department of Physical Chemistry, University of Santiago de Compostela, E-15706 Santiago \\ de Compostela, Spain \\ Henrik Koch ${ }^{\text {b) }}$ \\ Department of Chemistry, University of Southern Denmark, DK-5230 Odense M, Denmark
}

(Received 1 April 2002; accepted 21 May 2002)

\begin{abstract}
The first three-dimensional $a b$ initio intermolecular potential energy surface of the $\mathrm{Ar}-\mathrm{CO}$ van der Waals complex is calculated using the coupled cluster singles and doubles including connected triples model and the augmented correlation-consistent polarized valence quadruple zeta (aug-cc-pVQZ) basis set extended with a $(3 s 3 p 2 d 1 f 1 g)$ set of midbond functions. The three-dimensional surface is averaged over the three lowest vibrational states of CO. Rovibrational energies are calculated up to $50 \mathrm{~cm}^{-1}$ above the ground state, thus enabling comprehensive comparison between theory and available experimental data as well as providing detailed guidance for future spectroscopic investigations of higher-lying states. The experimental transitions are reproduced with a root-mean-square error of $0.13 \mathrm{~cm}^{-1}$, excluding states observed around $25 \mathrm{~cm}^{-1}$ above the ground state. The latter states are at variance with the experimentally deduced ordering.

(C) 2002 American Institute of Physics. [DOI: 10.1063/1.1493180]
\end{abstract}

\section{INTRODUCTION}

The Ar-CO complex can be considered a prototype van der Waals system, as witnessed by the extensive experimental $^{1-19}$ and theoretical ${ }^{20-31}$ work on its description. For example, the first paper, published in 1978 by Parker and Pack, ${ }^{20}$ used Ar-CO as a model system for the development of electron gas methods for calculating intermolecular potential energy surfaces (IPESs). A decade later, Tennyson, Miller, and Sutcliffe ${ }^{22}$ employed Ar-CO, aided by the IPES of Mirsky, ${ }^{21}$ as an example of a system that cannot be properly described by small-amplitude vibrations and near-rigid rotations, and therefore required development of new methodology for calculating rovibrational energies. Likewise, the first experimental observation of $\mathrm{Ar}-\mathrm{CO}$ was made in 1989 by De Piante, Campbell, and Buelow ${ }^{1}$ as part of work on developing sensitive equipment for spectroscopic investigations of weakly absorbing systems. Recently, with the number of observed transitions being on the order of $10^{3}, \mathrm{Ar}-\mathrm{CO}$ has been used as a reference system for novel design of spectrometers. ${ }^{18}$

Increasing attention, both theoretically and experimentally, has been focused on $\mathrm{Ar}-\mathrm{CO}$ during the last decade, arising, in part, from the prospects of eventually monitoring all the bound van der Waals states. Experimentally, the lower-lying levels comprising the ground state and the excited bending and stretching states are extensively character-

\footnotetext{
${ }^{a)}$ Electronic mail: bondo@slater.quifis.uv.es

b) Present address: Department of Physical Chemistry, Institute of Molecular Science, University of Valencia, E-46100 Burjassot, Valencia, Spain.
}

ized through the work of a number of authors, ${ }^{2-15}$ whereas observations of higher-lying states have only recently been reported by Scheele, Lehnig, and Havenith. ${ }^{16,17}$ On the theoretical side, a large part of the electronic structure methods, semiempirical $^{23,28,29,31}$ as well as ab initio, ${ }^{24-27,30}$ has been employed for calculating IPESs to assist in assigning experimental spectra. To this end, the most successful IPES is doubtlessly that computed by Jansen in $1994^{24}$ using the coupled pair functional (CPF) method and later semiempirically extrapolated to give the ECPF IPES by the same author in $1996 .{ }^{28}$ The CPF interaction energies were refitted with the aim of reproducing the lowest excited (bending) frequency, but the higher-lying levels are nevertheless of amazing accuracy. As an example, the rovibrational energies calculated from the ECPF IPES were of decisive importance for the assignments of the higher-lying states in Refs. 16 and 17.

The most accurate of the $a b$ initio IPESs are those of Toczyłowski and Cybulski, ${ }^{30}$ who used the coupled cluster singles and doubles including connected triples $[\operatorname{CCSD}(\mathrm{T})]^{32}$ model and two basis sets of triple-zeta quality differing only in additional sets of midbond functions. While these IPESs are comparable in accuracy to Jansen's for the lower states investigated, the experimental frequencies are not reproduced with the same precision for both basis sets, thus indicating that an even larger basis set may be needed. Although the differences are small, the bending frequency is best described with the larger basis set, while the stretching frequency is best described with the smaller. Moreover, no levels were reported above the excited stretching state.

In the most recent theoretical study, Gianturco and 
Paesani ${ }^{31}$ presented a semiempirical IPES obtained by a density functional theory-based model designated HHDSD. ${ }^{29} \mathrm{~A}$ novel feature of this IPES is that the CO bond was allowed to relax upon complex formation. Subsequently, neglecting diabatic couplings, this three-dimensional IPES was averaged over the lowest vibrational states of the free $\mathrm{CO}$ molecule. Encouraging results were reported for the rotational constants of the van der Waals ground state.

The experimental transitions reported by Scheele, Lehnig, and Havenith, ${ }^{16,17}$ although assigned on the basis of Jansen's energy levels, still pose a challenge to theory. All existing IPESs contradict the experimentally deduced level ordering around $25 \mathrm{~cm}^{-1}$ above the ground state. In an attempt to meet this challenge through ab initio methodology, we start out by noting that "the level of agreement between theory and experiment $[\cdots]$ is exceptional" ${ }^{33}$ when the coupled cluster IPES of Toczyłowski and Cybulski ${ }^{30}$ is employed for calculating the asymmetric component of the line shifts of CO diluted in Ar. However, the comparison of theory and experiment in Ref. 33 is incomplete, since calculation of the symmetric component, and therefore of the total line shift, requires a three-dimensional IPES. Consequently, we compute in this paper the three-dimensional Ar-CO IPES using the $\operatorname{CCSD}(\mathrm{T})$ model with a basis set selected from very strict convergence criteria. Subsequently, an averaging procedure analogous to that used by Gianturco and Paesani ${ }^{31}$ is invoked for calculating rovibrational energies up to $50 \mathrm{~cm}^{-1}$ above the ground state.

\section{THE VIBRATIONALLY ADIABATIC APPROXIMATION}

Let $\mathbf{r}$ be the vector of length $r$ from $\mathrm{C}$ to $\mathrm{O}, \mathbf{R}$ the vector of length $R$ from the center of mass of $\mathrm{CO}$ to $\mathrm{Ar}$, and $\theta$ the angle between $\mathbf{r}$ and $\mathbf{R}$. Hence, $\theta=0^{\circ}$ corresponds to linear $\mathrm{CO}-\mathrm{Ar}$ and $\theta=180^{\circ}$ corresponds to linear Ar-CO. Subscribing to the Born-Oppenheimer approximation, the potential (electronic) energy may be written as

$$
V(r, R, \theta)=V_{\mathrm{CO}}(r)+\Delta V(r, R, \theta),
$$

where $V_{\mathrm{CO}}(r)$ is the potential energy curve of the isolated CO molecule and $\Delta V(r, R, \theta)$ is the IPES. The constant contribution from Ar has been removed by a unitary transformation shifting the origin of energy.

Embedding a body-fixed coordinate system with the quantization $\left(z^{-}\right)$axis along $\mathbf{R}$, the nuclear Schrödinger equation may be written as ${ }^{34,35}$

$$
H \Psi_{l}^{J, p}(r, R, \theta, \alpha, \beta, \gamma)=E_{l}^{J, p} \Psi_{l}^{J, p}(r, R, \theta, \alpha, \beta, \gamma),
$$

after separation of the (nuclear) center of mass motion and noting that the eigenstates may be labeled by the total angular momentum quantum number $J=0,1,2, \ldots$ and the parity $p= \pm 1$. The eigenvalues are degenerate (in the absence of external fields) in the projection of the total angular momentum on the space-fixed $Z$ axis, characterized by the quantum number $M$, which is therefore not included in the notation. The index $l$ is merely a counter and $\alpha, \beta$, and $\gamma$ are the Euler angles for transforming between the space- and body-fixed coordinate frames. The rovibrational wave function is expanded in a basis set as

$$
\begin{aligned}
& \Psi_{l}^{J, p}(r, R, \theta, \alpha, \beta, \gamma) \\
& \quad=\sum_{\nu_{\mathrm{CO}}} \sum_{n j k} d_{\nu_{\mathrm{CO}}{ }^{n j k}}^{J, p, l} \widetilde{G}_{\nu_{\mathrm{CO}}}(r) G_{n}(R) F_{j k}^{J, p}(\theta, \alpha, \beta, \gamma),
\end{aligned}
$$

where $\widetilde{G}_{\nu_{\mathrm{CO}}}(r)$ is a purely vibrational (i.e., for vanishing angular momentum) eigenfunction of the isolated $\mathrm{CO}$ molecule, $G_{n}(R)$ is a Morse-type oscillator eigenfunction, and the angular basis functions are parity and total angular momentum eigenfunctions given by ${ }^{34}$

$$
\begin{aligned}
& F_{j k}^{J, p}(\theta, \alpha, \beta, \gamma) \\
& \quad=\frac{\Theta_{j, k}(\theta) \mathcal{D}_{M, k}^{J}(\alpha, \beta, \gamma)+p(-1)^{J} \Theta_{j,-k}(\theta) \mathcal{D}_{M,-k}^{J}(\alpha, \beta, \gamma)}{\sqrt{2\left(1+\delta_{k 0}\right)}} .
\end{aligned}
$$

Here, $\Theta_{j, k}(\theta)$ is an associated Legendre polynomial and $\mathcal{D}_{M, k}^{J}(\alpha, \beta, \gamma)$ is a rotation matrix element. ${ }^{36}$ The explicit form of the resulting secular Hamiltonian matrix can be inspected in Refs. 34 and 37.

For our present purposes, the most important feature is that the Hamiltonian couples the vibrational states of CO. Therefore, if applied in the form outlined above, the theory can be characterized as vibrationally diabatic. There are two terms in the Hamiltonian responsible for the diabatic coupling of the $\mathrm{CO}$ vibrational states: the three-dimensional IPES and the CO "rotational" constant depending on $r$ as $r^{-2}$. As is well known, the vibrational energy of the CO monomer is (at least) one order of magnitude larger than the energy of the intermolecular van der Waals modes, suggesting that the inter- and intramolecular nuclear motions may be considered essentially independent. Such decoupling of the equations, giving a vibrationally adiabatic approximation, can be achieved by simply neglecting all off-diagonal matrix elements of the IPES and $r^{-2}$ in the basis of the CO vibrational states. In this way, the IPES becomes effectively twodimensional and the vibrationally averaged rotational constants of the isolated CO molecule should be applied for calculating the rovibrational energies and wave functions. In the standard approach the IPES is assumed two-dimensional, fixing $r$ at some suitable value such as the equilibrium bond distance $r_{e}$. Thus, in the standard approach, there is no diabatic coupling due to the IPES by way of construction. A physically more reasonable procedure, used in this paper although far more elaborate in terms of computational cost, is to retain the $r$ dependence of the IPES and subsequently perform the dynamical calculations using the appropriately averaged IPES and rotational constant for each CO vibrational state. The standard approach utilizing an inherently adiabatic IPES has been used in all but the latest theoretical investigation of the $\mathrm{Ar}-\mathrm{CO}$ van der Waals complex in which Gianturco and Paesani, ${ }^{31}$ by means of a semiempirical density functional theory-based scheme for calculating IPESs, ${ }^{29}$ showed that the diabatic couplings due to the IPES are indeed negligible for bound state calculations in the three lowest vibrational states of $\mathrm{CO}$.

Adopting, therefore, the vibrationally adiabatic approximation and noting (from experiment) that $\mathrm{Ar}-\mathrm{CO}$ is a prolate near-symmetric rotor, the counter $l$ can be associated with 


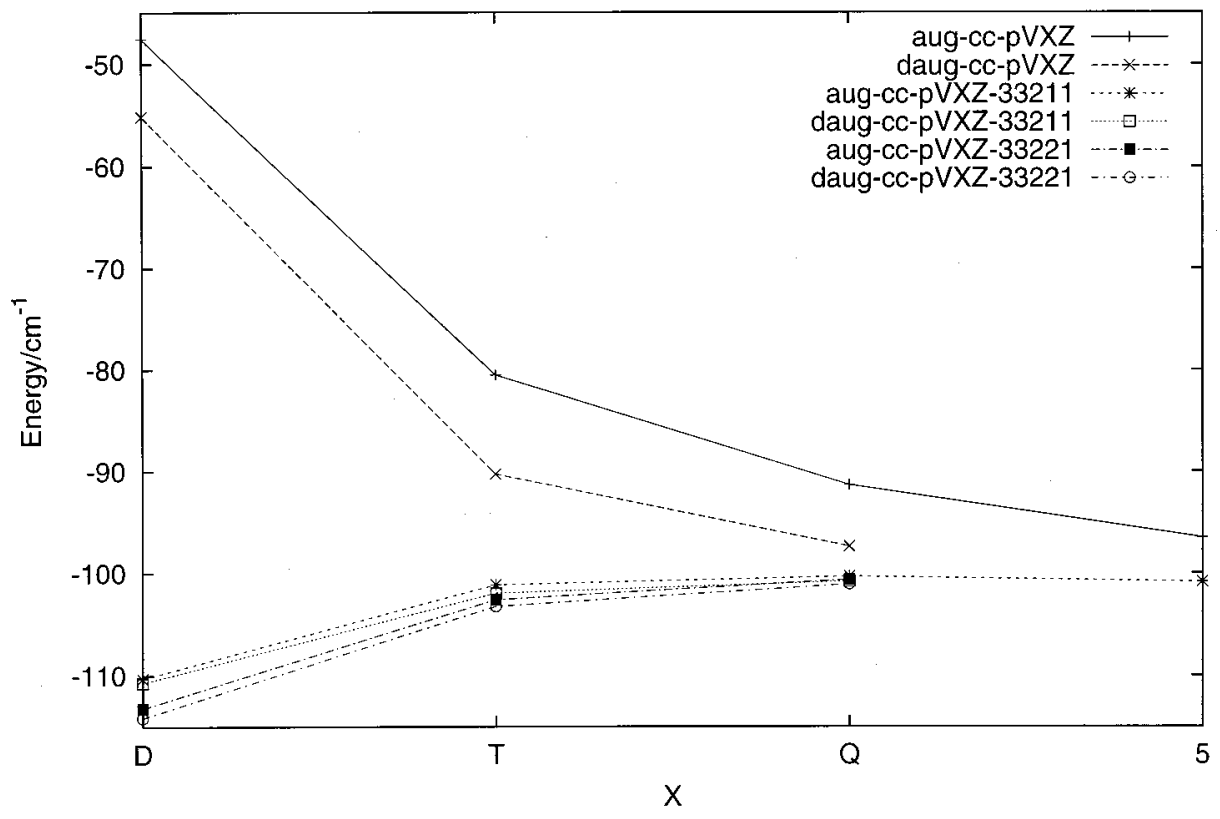

FIG. 1. Interaction energy at $r=r_{e}$ $=2.132$ bohr, $R=3.70 \AA$, and $\theta$ $=80^{\circ}$ as a function of the cardinal number of the basis set. three quantum numbers $\left(\nu_{\mathrm{CO}}, \nu_{\mathrm{vdW}}, K\right)$. While $\nu_{\mathrm{CO}}$ and $\nu_{\mathrm{vdW}}$ label the intra- and intermolecular vibrational modes, respectively, the approximate quantum number $K$ (equal to $K_{a}$ in the usual $J_{K_{a} K_{c}}$ notation of asymmetric rotors) represents the projection of the total angular momentum on the intermolecular $a$ axis. Thus, a set of energy levels $\left(\nu_{\mathrm{vdW}}, K\right)$, split into two components (for $K>0$ ) according to parity $p$ $= \pm 1$, can be calculated for each $\mathrm{CO}$ vibrational state $\nu_{\mathrm{CO}}$ and each total angular momentum quantum number $J$. The energy of a given state is then given by

$$
E_{\nu_{\mathrm{CO}}, \nu_{\mathrm{vdW}}, K}^{J, p}=E_{\nu_{\mathrm{CO}}}+\Delta E_{\nu_{\mathrm{CO}}, \nu_{\mathrm{vdW}}, K}^{J, p},
$$

where $E_{\nu_{\mathrm{CO}}}$ is the CO vibrational energy and $\Delta E_{\nu_{\mathrm{CO}}, \nu_{\mathrm{vdW}}, K}^{J, p}$ is the van der Waals mode energy. Transition frequencies are then obtained as simple energy differences.

Summarizing, the approximations that we invoke for calculating the rovibrational energy levels of the Ar-CO van der Waals complex are the Born-Oppenheimer approximation, the vibrationally adiabatic approximation, the use of a finite rovibrational basis set, and finally whatever approximations employed for modeling the IPES and $V_{\mathrm{CO}}(r)$.

\section{INTERMOLECULAR POTENTIAL ENERGY SURFACE}

\section{A. Method and basis set}

Without further testing, and based upon numerous successful applications to weakly bound systems (see, e.g., Refs. $30,38-42$ ), we employ the closed-shell $\operatorname{CCSD}(\mathrm{T}) \operatorname{model}^{32}$ in conjunction with variants of Dunning's correlation-consistent polarized basis sets ${ }^{43-46}$ for calculating the three-dimensional IPES of Ar-CO. The basis set superposition error is accounted for through the counterpoise correction scheme, ${ }^{47}$ and the electronic structure calculations are carried out with the DALTON program system. ${ }^{48}$

Toczyłowski and Cybulski ${ }^{30}$ used $\operatorname{CCSD}(\mathrm{T})$ with augcc-pVTZ extended with two different sets of midbond func- tions placed at $\mathbf{R} / 2$ relative to the center of mass of $\mathrm{CO}$. The two sets of midbond functions, $(3 s 3 p 2 d 1 f 1 g)^{49}$ and ( $3 s 3 p 2 d 2 f 1 g),{ }^{40}$ henceforth denoted 33211 and 33221, respectively, differ not only in size but also in the exponents (see the indicated references for details). While results of similar overall quality were obtained by Toczyłowski and Cybulski $^{30}$ with the aug-cc-pVTZ-33211 and aug-cc-pVTZ33221 basis sets, the smaller set describes the stretching vibration slightly better than the larger set which, in turn, yields improved results for the bending vibration. This may indicate that the quadruple-zeta basis set level is needed when the $r$ dependence of the IPES is included. Furthermore, a quadruple-zeta basis set is needed in order to describe the repulsive parts of the IPES.

In Fig. 1 we have plotted the interaction energy as a function of the cardinal number $(\mathrm{X}=\mathrm{D}, \mathrm{T}, \mathrm{Q}, 5)$ of the basis set for the nuclear conformation $r=r_{e}=2.132 \mathrm{bohr}, R$ $=3.70 \AA$, and $\theta=80^{\circ}$. The figure includes augmented as well as double augmented correlation-consistent polarized basis sets ${ }^{43-46}$ with and without midbond functions. The convergence pattern observed for 17 different nuclear conformations, involving three $\mathrm{CO}$ bond distances and probing all areas of the IPES, is similar to that of Fig. 1. This figure may therefore be considered globally representative, although the midbond functions are less effective in the repulsive regions. Noting that the difference between the two sets of midbond functions has nearly vanished at $\mathrm{X}=\mathrm{Q}$, and importing from Bak et al. ${ }^{50}$ the conclusion that $\operatorname{CCSD}(\mathrm{T})$ offers an accurate description of $\mathrm{CO}$ near the equilibrium bond distance at the quadruple-zeta basis set level, we employ the aug-cc-pVQZ33211 basis set for calculating the Ar-CO IPES.

All calculations are carried out using the frozen core approximation. However, Bak et al. ${ }^{50}$ stressed that their conclusion was valid only for calculations allowing all orbitals to contribute to the correlation energy. A test of the effect of freezing the core orbitals reveals a change in interaction energy of at most $0.9 \%$ (occurring on the repulsive wall) at the 
TABLE I. Equilibrium dissociation energy $D_{e}\left(\right.$ in $\mathrm{cm}^{-1}$ ), distance $R_{e}$ (in $\AA$ ), and angle $\theta_{e}$ (in degrees) from recent an initio and semiempirical IPESs at $r=r_{e}$.

\begin{tabular}{lllrrr}
\hline \hline Method & \multicolumn{1}{c}{ Basis set/comments } & Ref. & $D_{e}$ & $R_{e}$ & $\theta_{e}$ \\
\hline CPF & C,O: $6 s 5 p 3 d 1 f ;$ Ar: $7 s 6 p 3 d 1 f$ & 24 & 71 & 3.86 & 92 \\
MP2 & C,O: $3 s 2 p 2 d ;$ Ar:5s3p2d & 25 & 69 & 4.00 & 100 \\
MP4 & C,O:5s3p2d; Ar:7s4p2d1f & 26 & 108 & 3.75 & 80 \\
MP4 & C,O: $7 s 5 p 3 d ;$ Ar: $8 s 6 p 3 d ;$ mb:3s3p2d & 27 & 96 & 3.74 & 82 \\
ECPF & Extrapolated CPF & 28 & 109 & 3.68 & 97 \\
HHDSD & DFT/cc-pVQZ+empirical dispersion & 29 & 90 & 3.82 & 99 \\
CCSD(T) & aug-cc-pVTZ-33221 & 30 & 105 & 3.71 & 93 \\
CCSD(T) & aug-cc-pVTZ-33211 & 30 & 103 & 3.72 & 93 \\
CCSD(T) & aug-cc-pVQZ-33211 & This work & 102 & 3.72 & 93 \\
\hline \hline
\end{tabular}

a" $\mathrm{mb}$ " denotes a set of midbond functions.

aug-cc-pVQZ-33211 basis set level, and the frozen core approximation is thus justified for calculating the IPES.

In summary, the IPES of $\mathrm{Ar}-\mathrm{CO}$ is calculated at the counterpoise-corrected frozen core CCSD(T)/aug-cc-pVQZ33211 level. Since we are only interested in the lower vibrational states of $\mathrm{CO}\left(\nu_{\mathrm{CO}}=0,1,2\right)$ and following earlier work on the $\mathrm{He}-\mathrm{CO}$ complex, ${ }^{41,51}$ a grid is chosen for the bond distances $r=1.898,2.132$, and 2.134 bohr with additional points calculated for $r=2.050$ and 2.170 bohr. All 467 grid points calculated may be inspected in Ref. 52.

\section{B. Potential fit and vibrational averaging}

We use the same analytical form, originally suggested by Bukowski et al. ${ }^{53}$ for fitting the calculated grid points at fixed $r=1.898,2.132$, and 2.134 bohr as employed by Toczyłowski and Cybulski ${ }^{30}$ for their two-dimensional IPES. Every adjustable parameter is then assumed to be a quadratic function of $\left(r-r_{e}\right), r_{e}=2.132 \mathrm{bohr}$, and the remaining 60 $a b$ initio points calculated at $r=2.050$ and $2.170 \mathrm{bohr}$ serve as a test of the fitted potential. The total root-mean-square (rms) error, gauging also the 60 test points that were excluded in the fitting procedure, is $1.7 \mathrm{~cm}^{-1}$. Limiting the comparison to points of negative interaction energy (the bound region), the rms error reduces to $0.16 \mathrm{~cm}^{-1}$.

The equilibrium intermolecular geometry, $R_{e}$ and $\theta_{e}$, and dissociation energy, $D_{e}$, for various IPESs at $r=r_{e}$ are given in Table I. As could be anticipated from Fig. 1, the equilibrium parameters are rather similar for the three $\operatorname{CCSD}(\mathrm{T})$ IPESs. It is noteworthy, however, that the highly successful $\mathrm{ECPF}^{28}$ IPES yields the largest equilibrium dissociation energy of $109 \mathrm{~cm}^{-1}$, while the best $\operatorname{CCSD}(\mathrm{T})$ result is a significantly smaller $D_{e}$ value of about $102 \mathrm{~cm}^{-1}$. The barrier for rotation about the oxygen end of $\mathrm{CO}$ (i.e., the minimum for $\left.\theta=0^{\circ}\right)$ is $19.8 \mathrm{~cm}^{-1}$, and that for rotation about the carbon end $\left(\theta=180^{\circ}\right)$ is $30.5 \mathrm{~cm}^{-1}$. While the former is nearly identical to the $\mathrm{ECPF}^{28}$ barrier of $19.9 \mathrm{~cm}^{-1}$, the latter is some $15 \%$ larger (the ECPF barrier is 26.0 $\mathrm{cm}^{-1}$ ).

The vibrational matrix elements, $\Delta V_{\nu_{\mathrm{CO}}^{\prime}{ }_{\mathrm{CO}}}(R, \theta)$ $=\Delta V_{\nu_{\mathrm{CO}} \nu_{\mathrm{CO}}^{\prime}}(R, \theta)$, may be calculated with the aid of a Taylor expansion of $\Delta V(r, R, \theta)$ about $r_{e}$ as

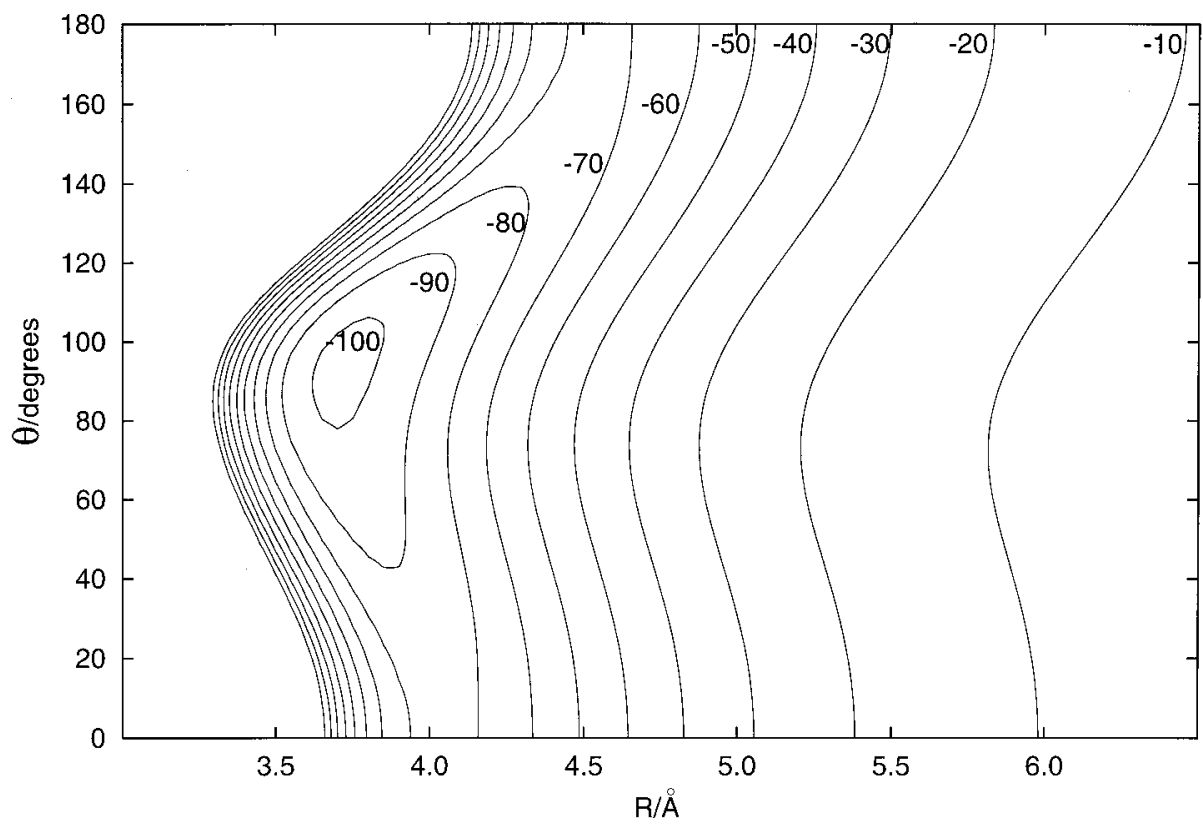

FIG. 2. Contours of the IPES averaged in the $\mathrm{CO}$ vibrational ground state. 


$$
\begin{aligned}
\Delta V_{\nu_{\mathrm{CO}}^{\prime} \nu_{\mathrm{CO}}}(R, \theta)= & \left\langle\widetilde{G}_{\nu_{\mathrm{CO}}^{\prime}}|\Delta V(r, R, \theta)| \widetilde{G}_{\nu_{\mathrm{CO}}}\right\rangle \\
= & \sum_{k=0}^{k_{\max }} \frac{1}{k !}\left(\frac{\partial^{k} \Delta V(r, R, \theta)}{\partial r^{k}}\right)_{r=r_{e}} \\
& \times\left\langle\widetilde{G}_{\nu_{\mathrm{CO}}^{\prime}}\left|\left(r-r_{e}\right)^{k}\right| \widetilde{G}_{\nu_{\mathrm{CO}}}\right\rangle,
\end{aligned}
$$

where the $\mathrm{CO}$ vibrational eigenfunctions $\widetilde{G}_{\nu_{\mathrm{CO}}}(r)$ are determined from the potential curve $V_{\mathrm{CO}}(r)$ for zero angular momentum. Following analogous work on the $\mathrm{He}-\mathrm{CO}$ complex, ${ }^{51}$ we deploy the empirical potential curve of Huxley and Murrel $^{54}$ (with $r_{e}=2.132$ bohr) for calculating $\widetilde{G}_{\nu_{\mathrm{CO}}}(r), \nu_{\mathrm{CO}}=0,1,2$, and the matrix elements of $\left(r-r_{e}\right)^{k}$ using the VIBROT module of the MOLCAS program suite. ${ }^{55}$ The Taylor series of the IPES was truncated at $k_{\max }=8$, as the matrix elements of $\left(r-r_{e}\right)^{k}$ in Eq. (6) are all smaller than $10^{-6} \mathrm{~cm}^{-1}$ for larger $k$.

Figure 2 plots contours of the $\Delta V_{00}$ surface. The $\mathrm{CO}$ ground-state IPES is seen to be rather flat, making possible large amplitude motions, even in the van der Waals ground state. Contour plots of the $\Delta V_{11}$ and $\Delta V_{22}$ surfaces show the same overall features, although they have slightly deeper minima.

In order to qualitatively confirm that diabatic couplings through the IPES are indeed negligible for bound state calculations, the elements of the vibrational matrix $\Delta V_{\nu_{\mathrm{CO}}^{\prime}{ }_{\mathrm{CO}}}\left(R, \theta=90^{\circ}\right)$ are plotted in Fig. 3. It should be noted that, since the vibrational wave functions are only determined up to a phase, the sign of the off diagonal coupling elements is arbitrary. In agreement with the findings of Gianturco and Paesani, ${ }^{31}$ the diabatic couplings are important only in regions that are inaccessible to the bound states.
A FORTRAN subroutine for generating the full threedimensional IPES as well as the vibrational matrix elements, using $\mathrm{CO}$ wave functions derived from the empirical potential curve, can be obtained from Ref. 52.

\section{ROVIBRATIONAL ENERGY LEVELS}

\section{A. Computational details}

The rovibrational energy levels and wave functions are calculated variationally with the program TRIATOM $^{37}$ using basis functions as outlined in Sec. II. The radial basis functions are chosen as Morse-type oscillator functions parametrized by the equilibrium distance, dissociation energy, and fundamental frequency of $7.55 \mathrm{bohr}, 0.4 \times 10^{-3}$ hartree, and $0.1 \times 10^{-3}$ hartree, respectively. The following nuclear masses and rotational constants are used: $\mathrm{m}\left({ }^{40} \mathrm{Ar}\right)$ $=39.962384 \mathrm{amu}, \quad \mathrm{m}\left({ }^{12} \mathrm{C}\right)=12.000000 \mathrm{amu}, \quad \mathrm{m}\left({ }^{16} \mathrm{O}\right)$ $=15.99491501 \mathrm{amu}, \quad \mathrm{B}_{0}=1.922528665 \mathrm{~cm}^{-1}, \quad \mathrm{~B}_{1}$ $=1.905024255 \mathrm{~cm}^{-1}$, and $\mathrm{B}_{2}=1.887519845 \mathrm{~cm}^{-1}$. Finally, transition frequencies of the isolated $\mathrm{CO}$ monomer are 2143.2711 and $4260.0621 \mathrm{~cm}^{-1}$ for the fundamental and overtone bands, respectively.

In order to further test the validity of the vibrationally adiabatic approximation, we calculate the energy of the three lowest van der Waals states in the $\mathrm{CO}$ ground state for $J$ $=0$ in two different ways. First, we use the two-dimensional $\Delta V_{00}(R, \theta)$ IPES and the rotational constant $\mathrm{B}_{0}$ given above. Second, we use the full three-dimensional potential, Eq. (1), with $V_{\mathrm{CO}}(r)$ equal to the empirical potential curve ${ }^{54}$ also used for the vibrational averaging. In both calculations, the intermolecular degrees of freedom are spanned by 15 radial and 15 angular basis functions. For the full threedimensional calculation, the $\mathrm{CO}$ stretch is spanned by 15

\begin{tabular}{|c|c|c|c|c|c|c|c|c|}
\hline \multirow{2}{*}{$\frac{\nu_{\mathrm{CO}}}{0}$} & \multirow{2}{*}{$\frac{\nu_{\mathrm{vdW}}}{0}$} & \multirow{2}{*}{$\begin{array}{c}K \\
1\end{array}$} & \multicolumn{2}{|c|}{ Experiment $^{\mathrm{a}}$} & \multirow{2}{*}{$\frac{\operatorname{CCSD}(\mathrm{T})^{\mathrm{b}}}{2.453}$} & \multirow[t]{2}{*}{$\operatorname{CCSD}(T)^{\mathrm{c}}$} & \multirow{2}{*}{$\frac{\mathrm{ECPF}^{\mathrm{d}}}{2.467}$} & \multirow{2}{*}{$\begin{array}{r}\text { HHDSD }^{\mathrm{e}} \\
2.478\end{array}$} \\
\hline & & & 2.416 & (12) & & & & \\
\hline & & 2 & 9.141 & (12) & 9.209 & & 9.296 & 9.291 \\
\hline & & 3 & 19.978 & (12) & 20.069 & & 20.261 & 20.021 \\
\hline & & 4 & 34.832 & (12) & 34.938 & & & 35.142 \\
\hline & 1 & 0 & 12.014 & (8) & 11.729 & 11.973 & 11.935 & 11.729 \\
\hline & & 1 & 17.146 & (8) & 17.051 & 17.391 & 16.881 & \\
\hline & 2 & 0 & 18.110 & (8) & 18.004 & 18.489 & 18.312 & \\
\hline \multirow[t]{10}{*}{1} & 0 & 1 & 2.393 & (12) & 2.422 & & & 2.442 \\
\hline & & 2 & 9.054 & (12) & 9.108 & & & 9.169 \\
\hline & & 3 & 19.790 & (12) & 19.860 & & & 19.964 \\
\hline & & 4 & 34.512 & (12) & 34.589 & & & 34.740 \\
\hline & 1 & 0 & 11.912 & (4) & 11.734 & & & 11.649 \\
\hline & & 1 & 17.028 & (6) & 17.015 & & & \\
\hline & 2 & 0 & 18.097 & (8) & 18.097 & & & 18.933 \\
\hline & & 1 & 26.187 & (10) & 24.033 & & & \\
\hline & 3 & 0 & 23.927 & (16) & 26.589 & & & \\
\hline & 5 & 0 & 36.765 & (17) & 36.074 & & & \\
\hline 2 & 0 & 1 & 2.372 & (15) & 2.391 & & & \\
\hline
\end{tabular}

TABLE II. Comparison of experimental and theoretical level origins $\left(\right.$ in $\mathrm{cm}^{-1}$ ) relative to the van der Waals ground state in each $\mathrm{CO}$ vibrational state.

${ }^{a}$ Experimental results from the reference in parenthesis.

${ }^{\mathrm{b}}$ This work; basis set aug-cc-pVQZ-33211.

${ }^{c}$ Reference 30; basis set aug-cc-pVTZ-33221. Values taken from the compilation in Ref. 17.

${ }^{\mathrm{d}}$ Reference 28. Values taken from the compilation in Ref. 17.

e Reference 31 . 


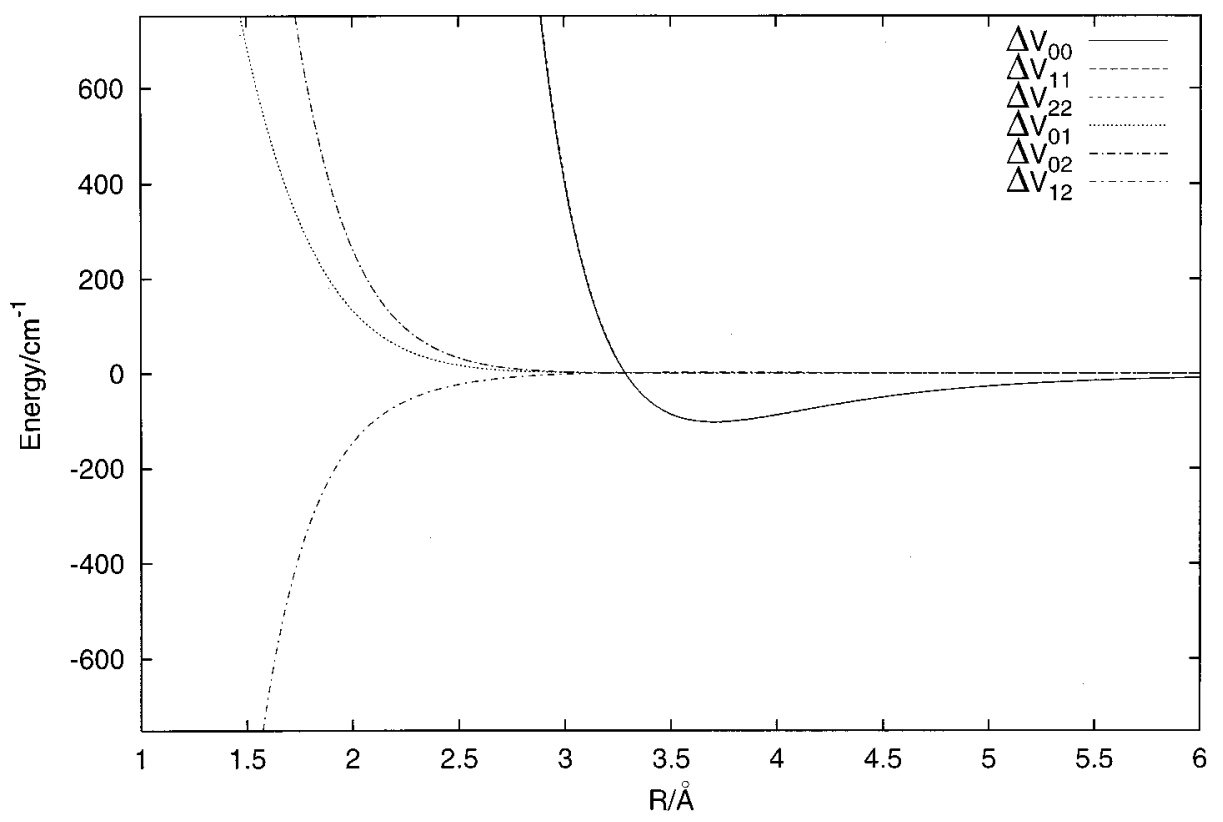

FIG. 3. Vibrational matrix elements of the three-dimensional IPES as a function of $R$ for $\theta=90^{\circ}$.
TABLE III. Spectroscopic constants $\left(\right.$ in $\mathrm{cm}^{-1}$ ) of the van der Waals ground state of Ar-CO for $\nu_{\mathrm{CO}}=0$.

\begin{tabular}{|c|c|c|c|}
\hline & Experiment $^{\mathrm{a}}$ & $\operatorname{CCSD}(\mathrm{T})^{\mathrm{b}}$ & $\operatorname{HHDSD}^{c}$ \\
\hline \multicolumn{4}{|l|}{$K=0:$} \\
\hline$\sigma$ & 0.0 & 0.0 & 0.0 \\
\hline$B$ & 0.0691008 & 0.0684479 & 0.0671 \\
\hline$D$ & $0.2032 \times 10^{-5}$ & $0.2007 \times 10^{-5}$ & $0.199 \times 10^{-5}$ \\
\hline$H$ & $-0.184 \times 10^{-9}$ & $-0.207 \times 10^{-9}$ & $-0.177 \times 10^{-9}$ \\
\hline$L$ & $-0.196 \times 10^{-12}$ & $-0.088 \times 10^{-12}$ & $-0.058 \times 10^{-12}$ \\
\hline \multicolumn{4}{|l|}{$K=1:$} \\
\hline$\sigma$ & 2.4161806 & 2.4526444 & 2.478 \\
\hline$B$ & 0.0687682 & 0.0681219 & 0.0667 \\
\hline$D$ & $0.2064 \times 10^{-5}$ & $0.2053 \times 10^{-5}$ & $0.200 \times 10^{-5}$ \\
\hline$H$ & $-0.314 \times 10^{-9}$ & $-0.293 \times 10^{-9}$ & $-0.277 \times 10^{-9}$ \\
\hline$L$ & $-0.186 \times 10^{-12}$ & $-0.151 \times 10^{-12}$ & $-0.104 \times 10^{-12}$ \\
\hline$b$ & 0.0021609 & 0.0021525 & 0.00211 \\
\hline$d$ & $0.01206 \times 10^{-5}$ & $0.01279 \times 10^{-5}$ & $0.00370 \times 10^{-5}$ \\
\hline$h$ & $0.0643 \times 10^{-9}$ & $0.0928 \times 10^{-9}$ & $0.0376 \times 10^{-9}$ \\
\hline$l$ & $0.1375 \times 10^{-12}$ & $0.0892 \times 10^{-12}$ & $0.470 \times 10^{-12}$ \\
\hline \multicolumn{4}{|l|}{$K=2:$} \\
\hline$\sigma$ & 9.1414616 & 9.2092852 & 9.291 \\
\hline$B$ & 0.0679368 & 0.0672849 & 0.0658 \\
\hline$D$ & $0.2629 \times 10^{-5}$ & $0.2668 \times 10^{-5}$ & $0.255 \times 10^{-5}$ \\
\hline$H$ & $-0.812 \times 10^{-9}$ & $-0.967 \times 10^{-9}$ & $-0.720 \times 10^{-9}$ \\
\hline$L$ & $-0.656 \times 10^{-12}$ & $-0.466 \times 10^{-12}$ & $-0.043 \times 10^{-12}$ \\
\hline$d$ & $0.78435 \times 10^{-6}$ & $0.88578 \times 10^{-6}$ & $0.920 \times 10^{-6}$ \\
\hline$h$ & $0.10477 \times 10^{-8}$ & $0.12472 \times 10^{-8}$ & $0.0896 \times 10^{-8}$ \\
\hline$l$ & $0.8747 \times 10^{-12}$ & $0.7738 \times 10^{-12}$ & $0.0115 \times 10^{-12}$ \\
\hline \multicolumn{4}{|l|}{$K=3:$} \\
\hline$\sigma$ & 19.978320 & 20.068958 & 20.021 \\
\hline$B$ & 0.0666914 & 0.066039 & 0.0645 \\
\hline$D$ & $0.3816 \times 10^{-5}$ & $0.3950 \times 10^{-5}$ & $0.342 \times 10^{-5}$ \\
\hline$H$ & $-1.970 \times 10^{-9}$ & $-1.161 \times 10^{-9}$ & $-1.540 \times 10^{-9}$ \\
\hline$L$ & $-0.121 \times 10^{-10}$ & $-0.168 \times 10^{-10}$ & $-0.070 \times 10^{-10}$ \\
\hline$h$ & $0.2097 \times 10^{-8}$ & $0.2265 \times 10^{-8}$ & $0.179 \times 10^{-8}$ \\
\hline$l$ & $0.0693 \times 10^{-10}$ & $0.0684 \times 10^{-10}$ & $0.0448 \times 10^{-10}$ \\
\hline$m$ & $0.3518 \times 10^{-13}$ & $0.4781 \times 10^{-13}$ & $0.195 \times 10^{-13}$ \\
\hline
\end{tabular}

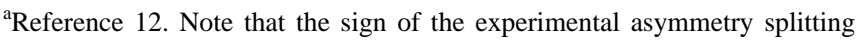
has been reversed for $K=2$ due to difference in the definition of e- and f-components.

${ }^{\mathrm{b}}$ This work.

${ }^{c}$ Reference 31 . additional Morse-type oscillator functions parametrized by $2.05 \mathrm{bohr}, 0.45$ hartree, and 0.009 hartree for the equilibrium distance, dissociation energy, and fundamental frequency, respectively. The ground-state energy obtained from the full three dimensional calculation is $-78.990921 \mathrm{~cm}^{-1}$ and that from the vibrationally adiabatic calculation is -78.989975 $\mathrm{cm}^{-1}$. Hence, diabatic effects alter the van der Waals groundstate energy by less than $10^{-3} \mathrm{~cm}^{-1}$. According to the full three-dimensional calculation, the lowest excited van der Waals states (bending and stretching, respectively) lie 11.729154 and $18.004368 \mathrm{~cm}^{-1}$ above the ground state. These values are only marginally different (2-3 $\times 10^{-4} \mathrm{~cm}^{-1}$ ) from the analogous two-dimensional results, and the vibrationally adiabatic approximation is therefore valid, as also suggested by Fig. 3 (see also Sec. III B).

Vibrationally adiabatic calculations are then performed with 50 radial and 100 angular basis functions for $J$ $=0,1,2, \ldots, 11$ and $\nu_{\mathrm{CO}}=0,1,2$. The calculations are carried out using the two-step procedure described in Ref. 56. In the first step, only the diagonal Coriolis coupling (i.e., between states of equal K) is included. Subsequently, the 1000 states of lowest energy from the first step are used in the second step to diagonalize the Hamiltonian, including the offdiagonal Coriolis coupling terms.

The van der Waals ground-state energies thus obtained are $-78.989975,-79.401372$, and $-79.755155 \mathrm{~cm}^{-1}$ for $\nu_{\mathrm{CO}}=0,1$, and 2 . Hence, the origins are shifted by -0.4114 and $-0.7652 \mathrm{~cm}^{-1}$ for the CO fundamental and overtone band, respectively. These results differ by $6 \%$ and $13 \%$, respectively, from the experimentally deduced shifts of $-0.4377 \mathrm{~cm}^{-1}$ for the fundamental ${ }^{12}$ and $-0.8778 \mathrm{~cm}^{-1}$ for the overtone band. ${ }^{15}$ It must be stressed that these shifts are not directly observable quantities; for a proper discussion of observable shifts of rovibrational CO lines, see Ref. 33. In conjunction with the contours of Fig. 2, the ground-state energy verifies that the $\mathrm{Ar}-\mathrm{CO}$ is a very floppy molecule with large radial as well as angular extent of the ground state. For 


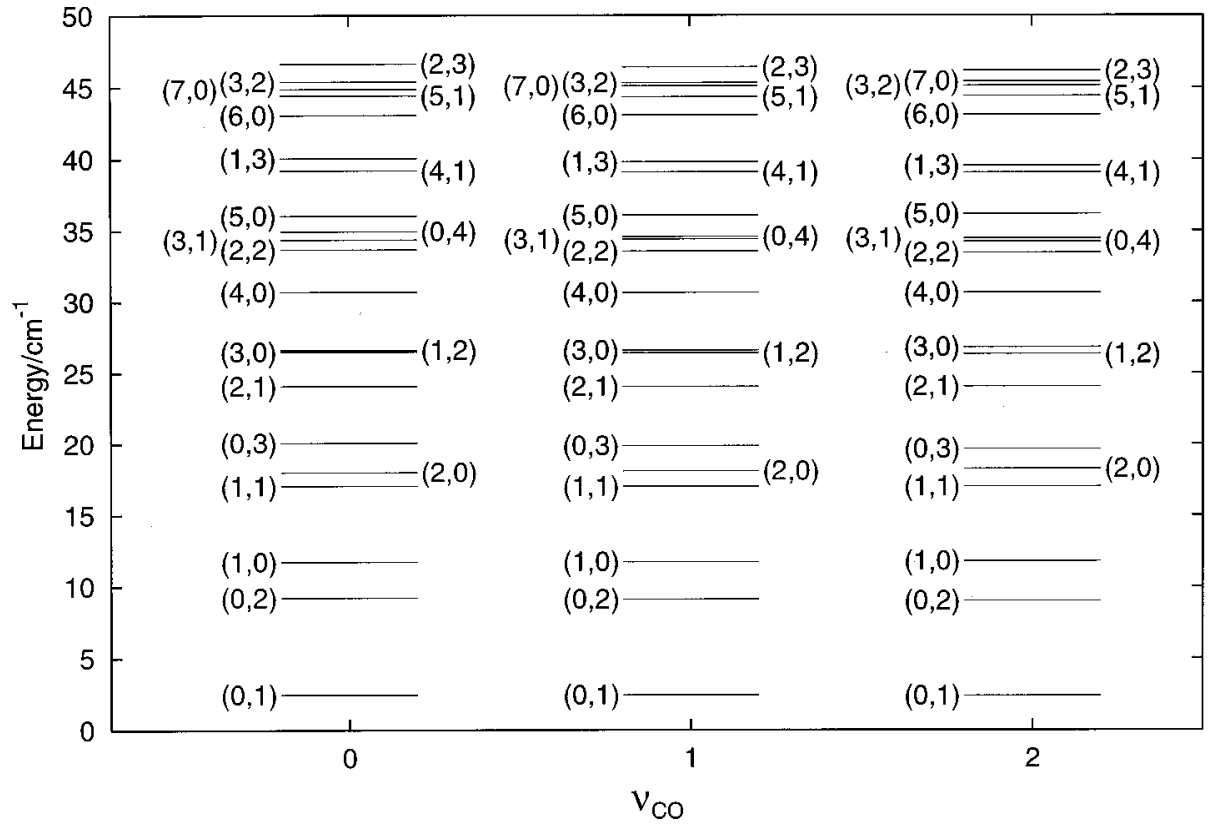

FIG. 4. Theoretical energy levels denoted by $\left(\nu_{\mathrm{vdW}}, K\right)$ for $\nu_{\mathrm{CO}}=0,1,2$. The quantity plotted is the level origin as obtained by fitting to Eq. (7).

$J=0$, the total number of vibrational states contained in the averaged potentials is 19 , somewhat fewer than the 23 vibrational states encountered in the ECPF IPES. ${ }^{28}$

A computer program is available ${ }^{52}$ for calculating spectra and printing tables of the assigned theoretical energies.

\section{B. Energy levels and Coriolis couplings}

Following Hepp et al., ${ }^{12}$ we fit the theoretical rovibrational energies to an expression derived from the traditional perturbative treatment of vibration-rotation coupling as

$$
E=\sigma+B Y-D Y^{2}+H Y^{3}+L Y^{4} \pm \frac{1}{2} S,
$$

for fixed values of the quantum numbers $\left(\nu_{\mathrm{CO}}, \nu_{\mathrm{vdW}}, K\right)$. The $-\operatorname{sign}$ is chosen for e-components for which $p(-1)^{J}$ $=+1$, whereas the + sign is used for f-components for which $p(-1)^{J}=-1$. In Eq. (7), $\sigma$ is the level origin relative to the van der Waals ground state of the appropriate $\mathrm{CO}$ vibrational state, $B$ is the rotational constant, $D$ is the centrifugal distortion constant, etc., and $Y=J(J+1)-K^{2}$. The asymmetry splitting is given by

$S=\left\{\begin{array}{l}0 \quad \text { for } K=0, \\ \quad b Z+d Z[J(J+1)]+h Z[J(J+1)]^{2}+l Z[J(J+1)]^{3} \\ \quad \text { for } K=1, \\ d Z+h Z[J(J+1)]+l Z[J(J+1)]^{2} \quad \text { for } K=2, \\ h Z+l Z[J(J+1)]+m Z[J(J+1)]^{2} \quad \text { for } K=3,\end{array}\right.$

where $Z=(J+K) ! /(J-K)$ !

While Eq. (7) offers an adequate representation (errors less than $10^{-5} \mathrm{~cm}^{-1}$ ) of the lower levels, it is generally less suitable (errors up to $10^{-1} \mathrm{~cm}^{-1}$ ) for the higher vibrational and rotational van der Waals levels. Our main purpose, therefore, is to obtain an approximate origin for each level $\left(\nu_{\mathrm{CO}}, \nu_{\mathrm{vdW}}, K\right)$ in order to facilitate comparison of theoretical and experimental level stacks. Comparison of spectro-
TABLE IV. Spectroscopic constants (in $\mathrm{cm}^{-1}$ ) of the van der Waals ground state of $\mathrm{Ar}-\mathrm{CO}$ for $\nu_{\mathrm{CO}}=1$.

\begin{tabular}{|c|c|c|c|}
\hline & Experiment $^{\mathrm{a}}$ & $\operatorname{CCSD}(T)^{b}$ & $\mathrm{HHDSD}^{\mathrm{c}}$ \\
\hline \multicolumn{4}{|l|}{$K=0$ : } \\
\hline$\sigma$ & 0.0 & 0.0 & 0.0 \\
\hline$B$ & 0.0691018 & 0.0684325 & 0.0675 \\
\hline$D$ & $0.2041 \times 10^{-5}$ & $0.1997 \times 10^{-5}$ & $0.196 \times 10^{-5}$ \\
\hline$H$ & $-0.108 \times 10^{-9}$ & $-0.208 \times 10^{-9}$ & $-0.174 \times 10^{-9}$ \\
\hline$L$ & $-0.261 \times 10^{-12}$ & $-0.066 \times 10^{-12}$ & $-0.046 \times 10^{-12}$ \\
\hline \multicolumn{4}{|l|}{$K=1:$} \\
\hline$\sigma$ & 2.3928 & 2.4221 & 2.442 \\
\hline$B$ & 0.0687587 & 0.0681087 & 0.0671 \\
\hline$D$ & $0.1971 \times 10^{-5}$ & $0.2036 \times 10^{-5}$ & $0.195 \times 10^{-5}$ \\
\hline$H$ & $-0.526 \times 10^{-9}$ & $-0.292 \times 10^{-9}$ & $-0.223 \times 10^{-9}$ \\
\hline$L$ & $-0.010 \times 10^{-12}$ & $-0.126 \times 10^{-12}$ & $-0.078 \times 10^{-12}$ \\
\hline$b$ & 0.0021675 & 0.0021538 & 0.00209 \\
\hline$d$ & $0.0224 \times 10^{-5}$ & $0.0119 \times 10^{-5}$ & $0.0022 \times 10^{-5}$ \\
\hline$h$ & $-0.215 \times 10^{-9}$ & $0.090 \times 10^{-9}$ & $0.037 \times 10^{-9}$ \\
\hline l & $0.342 \times 10^{-12}$ & $0.070 \times 10^{-12}$ & $0.029 \times 10^{-12}$ \\
\hline \multicolumn{4}{|l|}{$K=2$ : } \\
\hline$\sigma$ & 9.0536 & 9.1077 & 9.169 \\
\hline$B$ & 0.0679390 & 0.0672819 & 0.0663 \\
\hline$D$ & $0.2647 \times 10^{-5}$ & $0.2633 \times 10^{-5}$ & $0.243 \times 10^{-5}$ \\
\hline$H$ & $-0.850 \times 10^{-9}$ & $-0.917 \times 10^{-9}$ & $-0.662 \times 10^{-9}$ \\
\hline$L$ & $-0.523 \times 10^{-12}$ & $-0.517 \times 10^{-12}$ & $-0.123 \times 10^{-12}$ \\
\hline$d$ & $0.819 \times 10^{-6}$ & $0.859 \times 10^{-6}$ & $0.762 \times 10^{-6}$ \\
\hline$h$ & $0.0975 \times 10^{-8}$ & $0.1176 \times 10^{-8}$ & $0.0730 \times 10^{-8}$ \\
\hline l & $0.985 \times 10^{-12}$ & $0.843 \times 10^{-12}$ & $0.015 \times 10^{-12}$ \\
\hline \multicolumn{4}{|l|}{$K=3$ : } \\
\hline$\sigma$ & 19.7901 & 19.8598 & 19.964 \\
\hline$B$ & 0.066683 & 0.0660488 & 0.0650 \\
\hline$D$ & $0.382 \times 10^{-5}$ & $0.366 \times 10^{-5}$ & $0.319 \times 10^{-5}$ \\
\hline$H$ & $-0.020 \times 10^{-7}$ & $-0.027 \times 10^{-7}$ & $-0.007 \times 10^{-7}$ \\
\hline$L$ & $-0.096 \times 10^{-10}$ & $-0.096 \times 10^{-10}$ & $-0.055 \times 10^{-10}$ \\
\hline$h$ & $0.160 \times 10^{-8}$ & $0.198 \times 10^{-8}$ & $0.146 \times 10^{-8}$ \\
\hline$l$ & $0.1554 \times 10^{-10}$ & $0.066 \times 10^{-10}$ & $0.053 \times 10^{-10}$ \\
\hline$m$ & & $0.035 \times 10^{-12}$ & \\
\hline
\end{tabular}

${ }^{a}$ Reference 12 . Note that the sign of the experimental asymmetry splitting has been reversed for $K=2$ due to difference in the definition of e- and f-components.

${ }^{\mathrm{b}}$ This work.

${ }^{\mathrm{c}}$ Reference 31 . 
TABLE V. Spectroscopic constants (in $\mathrm{cm}^{-1}$ ) of the van der Waals ground state of $\mathrm{Ar}-\mathrm{CO}$ for $\nu_{\mathrm{CO}}=2$.

\begin{tabular}{ccc}
\hline \hline & Experiment $^{\mathrm{a}}$ & \multicolumn{1}{c}{$\operatorname{CCSD}(\mathrm{T})^{\mathrm{b}}$} \\
\hline$K=0:$ & & \\
$\sigma$ & 0.0 & 0.0 \\
$B$ & 0.0691065 & 0.0683983 \\
$D$ & $0.2039 \times 10^{-5}$ & $-0.201 \times 10^{-9}$ \\
$H$ & $-0.146 \times 10^{-9}$ & $-0.071 \times 10^{-12}$ \\
$L$ & $-0.196 \times 10^{-12}$ & \\
$K=1:$ & & 2.3911 \\
$\sigma$ & 2.3715 & 0.0680782 \\
$B$ & 0.0687348 & $0.2014 \times 10^{-5}$ \\
$D$ & $0.1925 \times 10^{-5}$ & $-0.282 \times 10^{-9}$ \\
$H$ & $-0.430 \times 10^{-9}$ & 0.0021484 \\
$L$ & $-0.186 \times 10^{-12}$ & $0.0106 \times 10^{-5}$ \\
$b$ & 0.0021974 & $0.080 \times 10^{-9}$ \\
$d$ & $0.0133 \times 10^{-5}$ & $0.0571 \times 10^{-12}$ \\
$h$ & $-0.080 \times 10^{-9}$ & \\
$l$ & $0.1375 \times 10^{-12}$ & \\
\hline \hline
\end{tabular}

${ }^{\mathrm{a}}$ Reference 15 .

${ }^{\mathrm{b}}$ This work.

scopic constants will be performed only for the van der Waals ground state in Sec. IV C.

Table II compares experimental and theoretical level origins. For the $(0,1,0)$ [bending] level, it should be stressed that the ECPF result compares favorably with experiment by way of construction; the ECPF surface was fitted so as to reproduce this level. ${ }^{28}$ The increased discrepancy between theory and experiment for the $(0,1,0)$ level when the basis set is increased may be an indication of incomplete description of dynamical correlation effects with the $\operatorname{CCSD}(\mathrm{T})$ model. For the stretching level $(0,2,0)$, on the other hand, the discrepancy decreases with increasing basis set as expected. Furthermore, the origin of the $(1,2,0)$ level is identical to that deduced experimentally. We also note that the $\left(\nu_{\mathrm{CO}}, 1,1\right)$ levels are excellently described with the vibrationally adiabatic $\operatorname{CCSD}(\mathrm{T})$ surfaces. A rather peculiar but not entirely unexpected feature is that the theoretical $(1,2,1)$ and $(1,3,0)$ levels evidently are interchanged compared to experiment, seemingly indicating that the higher-energy region is poorly described with the CCSD(T) IPES. However, the $(1,5,0)$ level, which is located above the $(1,2,1)$ and $(1,3,0)$ levels, compares rather well with experiment, thereby providing evidence that the IPES is in fact reasonably accurate also in the higher-energy regions. We shall return to this point in Sec. IV D.

A complete picture of the energy levels up to $50 \mathrm{~cm}^{-1}$ above the appropriate van der Waals ground state as obtained with the vibrationally averaged $\operatorname{CCSD}(\mathrm{T}) / \mathrm{aug}$-cc-pVQZ-

TABLE VI. Error statistics (in $\mathrm{cm}^{-1}$ ) for transitions not involving excitation of the $\mathrm{CO}$ vibrational mode; $N$ is the number of lines included from a given branch. Experimental results are taken from indicated references.

\begin{tabular}{cccrccc}
\hline \hline Transition & \multirow{2}{*}{ Ref. } & Branch & $N$ & $\Delta_{\min }$ & $\Delta_{\max }$ & $\Delta_{\text {rms }}$ \\
\hline$(0,1,0) \leftarrow(0,0,0)$ & \multirow{2}{*}{11} & $P$ & 8 & 0.2639 & 0.2835 & 0.2767 \\
& & $R$ & 6 & 0.2870 & 0.2894 & 0.2886 \\
$(0,2,0) \leftarrow(0,0,0)$ & \multirow{2}{*}{14} & $R$ & 11 & 0.1036 & 0.1295 & 0.1177 \\
\hline \hline
\end{tabular}

33211 IPES is displayed in Fig. 4. Only minor qualitative differences are observed going from $\nu_{\mathrm{CO}}=0$ to 2 , such as the splitting of the near-degenerate $\left(\nu_{\mathrm{CO}}, 3,0\right)$ and $\left(\nu_{\mathrm{CO}}, 1,2\right)$ levels with increasing $\mathrm{CO}$ vibrational quantum number. For all practical purposes, the van der Waals ground-state levels $\left(\nu_{\mathrm{CO}}, 0, \mathrm{~K}\right)$ are largely unaffected by Coriolis couplings. Even the $\left(\nu_{\mathrm{CO}}, 0,4\right)$ level, which is part of a cluster of close-lying levels around $35 \mathrm{~cm}^{-1}$, experiences only weak Coriolis couplings. Nevertheless, for the $(0,0,4)$ level these couplings are sufficiently strong to interchange the ordering of the e- and fcomponents at $J=8$, a feature that renders the fitting to Eq. (7) quite difficult. Interestingly, this does not happen for $\nu_{\mathrm{CO}}=1,2$. Whereas the $\left(\nu_{\mathrm{CO}}, 1,0\right)$ level is only slightly coupled, the e-component of $\left(\nu_{\mathrm{CO}}, 1,1\right)$ is strongly coupled to $\left(\nu_{\mathrm{CO}}, 2,0\right)$, as is also well documented experimentally. ${ }^{6,8}$ Strong Coriolis couplings are also found in the pairs $\left(\nu_{\mathrm{CO}}, 2,1\right),\left(\nu_{\mathrm{CO}}, 3,0\right)$ and $\left(\nu_{\mathrm{CO}}, 3,0\right),\left(\nu_{\mathrm{CO}}, 1,2\right)$ of the level cluster around $25 \mathrm{~cm}^{-1}$. The coupling between $\left(\nu_{\mathrm{CO}}, 2,1\right)$ and $\left(\nu_{\mathrm{CO}}, 3,0\right)$ was anticipated in the recent experimental work of Scheele, Lehnig, and Havenith. ${ }^{16}$ By contrast, only weak coupling between the $\left(\nu_{\mathrm{CO}}, 2,1\right)$ and $\left(\nu_{\mathrm{CO}}, 1,2\right)$ levels is encountered theoretically. The next level, $\left(\nu_{\mathrm{CO}}, 4,0\right)$, is entirely uncoupled and the $\left(\nu_{\mathrm{CO}}, 5,0\right)$ level from the cluster around $35 \mathrm{~cm}^{-1}$ is only very weakly coupled to the e-component of the $\left(\nu_{\mathrm{CO}}, 3,1\right)$ level, in reasonable agreement with experimental findings. ${ }^{17}$ Both e- and f-components of the latter are, in turn, coupled to those of the $\left(\nu_{\mathrm{CO}}, 2,2\right)$ level. The pair $\left(\nu_{\mathrm{CO}}, 4,1\right)$ and $\left(\nu_{\mathrm{CO}}, 1,3\right)$ at $40 \mathrm{~cm}^{-1}$ does not show Coriolis coupling. Finally, while the components of the remaining levels of the cluster around $45 \mathrm{~cm}^{-1}$ readily couple among each other, the $\left(\nu_{\mathrm{CO}}, 7,0\right)$ level is remarkably independent of its neighbors.

\section{The van der Waals ground state}

In this section, we pay special attention to the van der Waals ground state and the rotational sublevels, since they participate in a large fraction of the vast amount of rovibrational transitions reported in the literature. We would like to stress, however, that pure rotational spectra of van der Waals complexes are extremely difficult to reproduce, not to mention predict, theoretically, unless experimental data are explicitly included when fitting the $a b$ initio IPES, see, e.g., Ref. 38. As expected, therefore, our theoretical rotational spectra are of the same overall quality as those of Toczyłowski and Cybulski. ${ }^{30}$

The results of fitting the van der Waals ground-state energies for $\nu_{\mathrm{CO}}=0,1,2$ and $K=0,1,2,3$ to Eq. (7) are reported in Tables III-V along with available experimental data and the semiempirical results of Gianturco and Paesani. ${ }^{31}$ The errors of the $\operatorname{CCSD}(\mathrm{T})$ rotational constants $(B)$ are nearly constant at $1 \%$, whereas those obtained from the HHDSD IPESs vary from $2.3 \%$ to $3.3 \%$. The discrepancies of the centrifugal distortion constants $(D)$ are less regular and range from $0.5 \%$ to $4.6 \%$ for $\operatorname{CCSD}(\mathrm{T})$ and from $1.1 \%$ to $10 \%$ for HHDSD. With the exception of the $b$ constant entering the asymmetry splitting term of the $K=1$ levels, the remaining higher-order constants are generally calculated with much larger percent-wise errors. 
TABLE VII. Error statistics (in $\mathrm{cm}^{-1}$ ) for transitions in the CO fundamental band; $N$ is the number of lines included from a given branch. Experimental results are taken from indicated references.

\begin{tabular}{|c|c|c|c|c|c|c|}
\hline Transition & Ref. & Branch & $N$ & $\Delta_{\min }$ & $\Delta_{\max }$ & $\Delta_{\mathrm{rms}}$ \\
\hline \multirow[t]{3}{*}{$(1,0,1) \leftarrow(0,0,0)$} & 2 & $P$ & 10 & 0.0596 & 0.0719 & 0.0658 \\
\hline & & $Q$ & 11 & 0.0543 & 0.0565 & 0.0556 \\
\hline & & $R$ & 11 & 0.0428 & 0.0554 & 0.0494 \\
\hline \multirow[t]{3}{*}{$(1,0,0) \leftarrow(0,0,1)$} & 2 & $P$ & 11 & 0.0003 & 0.0075 & 0.0044 \\
\hline & & $Q$ & 11 & 0.0095 & 0.0122 & 0.0107 \\
\hline & & $R$ & 10 & 0.0139 & 0.0286 & 0.0208 \\
\hline \multirow[t]{6}{*}{$(1,0,2) \leftarrow(0,0,1)^{\mathrm{a}}$} & 2 & $P_{1}$ & 9 & 0.0497 & 0.0583 & 0.0544 \\
\hline & & $P_{2}$ & 9 & 0.0499 & 0.0605 & 0.0553 \\
\hline & & $Q_{1}$ & 10 & 0.0449 & 0.0462 & 0.0457 \\
\hline & & $Q_{2}$ & 10 & 0.0446 & 0.0460 & 0.0455 \\
\hline & & $R_{1}$ & 10 & 0.0296 & 0.0433 & 0.0369 \\
\hline & & $R_{2}$ & 10 & 0.0314 & 0.0432 & 0.0375 \\
\hline \multirow[t]{6}{*}{$(1,0,1) \leftarrow(0,0,2)^{\mathrm{b}}$} & 2 & $P_{1}$ & 10 & 0.0004 & 0.0106 & 0.0062 \\
\hline & & $P_{2}$ & 10 & 0.0023 & 0.0103 & 0.0067 \\
\hline & & $Q_{1}$ & 10 & 0.0128 & 0.0142 & 0.0136 \\
\hline & & $Q_{2}$ & 10 & 0.0137 & 0.0145 & 0.0141 \\
\hline & & $R_{1}$ & 9 & 0.0169 & 0.0257 & 0.0220 \\
\hline & & $R_{2}$ & 9 & 0.0174 & 0.0315 & 0.0247 \\
\hline \multirow[t]{6}{*}{$(1,0,3) \leftarrow(0,0,2)^{\mathrm{a}}$} & 2 & $P_{1}$ & 7 & 0.0368 & 0.0560 & 0.0453 \\
\hline & & $P_{2}$ & 7 & 0.0361 & 0.0390 & 0.0381 \\
\hline & & $Q_{1}$ & 9 & 0.0318 & 0.0600 & 0.0405 \\
\hline & & $Q_{2}$ & 9 & 0.0102 & 0.0317 & 0.0272 \\
\hline & & $R_{1}$ & 9 & 0.0224 & 0.0278 & 0.0248 \\
\hline & & $R_{2}$ & 9 & 0.0209 & 0.0278 & 0.0240 \\
\hline \multirow[t]{6}{*}{$(1,0,2) \leftarrow(0,0,3)^{\mathrm{b}}$} & 2 & $P_{1}$ & 9 & 0.0005 & 0.0077 & 0.0047 \\
\hline & & $P_{2}$ & 9 & 0.0006 & 0.0077 & 0.0044 \\
\hline & & $Q_{1}$ & 9 & 0.0116 & 0.0135 & 0.0127 \\
\hline & & $Q_{2}$ & 9 & 0.0111 & 0.0127 & 0.0122 \\
\hline & & $R_{1}$ & 8 & 0.0170 & 0.0282 & 0.0231 \\
\hline & & $R_{2}$ & 8 & 0.0169 & 0.0265 & 0.0223 \\
\hline \multirow[t]{2}{*}{$(1,1,0) \leftarrow(0,0,0)$} & 4 & $P$ & 9 & 0.1355 & 0.1502 & 0.1437 \\
\hline & & $R$ & 11 & 0.1541 & 0.1610 & 0.1585 \\
\hline \multirow[t]{4}{*}{$(1,1,1) \leftarrow(0,0,1)^{\mathrm{a}}$} & 6 & $P_{1}$ & 9 & 0.0001 & 0.0434 & 0.0235 \\
\hline & & $P_{2}$ & 10 & 0.0003 & 0.0119 & 0.0069 \\
\hline & & $R_{1}$ & 10 & 0.0003 & 0.0444 & 0.0212 \\
\hline & & $R_{2}$ & 10 & 0.0174 & 0.0239 & 0.0215 \\
\hline \multirow[t]{4}{*}{$(1,0,4) \leftarrow(0,0,3)^{\mathrm{a}}$} & 7 & $Q_{1}$ & 8 & 0.0003 & 0.0155 & 0.0094 \\
\hline & & $Q_{2}$ & 8 & 0.0049 & 0.1053 & 0.0452 \\
\hline & & $R_{1}$ & 8 & 0.0025 & 0.1181 & 0.0517 \\
\hline & & $R_{2}$ & 8 & 0.0001 & 0.0157 & 0.0083 \\
\hline \multirow[t]{4}{*}{$(1,0,3) \leftarrow(0,0,4)^{\mathrm{b}}$} & 7 & $P_{1}$ & 8 & 0.0010 & 0.1376 & 0.0809 \\
\hline & & $P_{2}$ & 8 & 0.0039 & 0.1294 & 0.0551 \\
\hline & & $Q_{1}$ & 8 & 0.0012 & 0.0230 & 0.0123 \\
\hline & & $Q_{2}$ & 8 & 0.0030 & 0.3181 & 0.1569 \\
\hline \multirow[t]{2}{*}{$(1,0,0) \leftarrow(0,1,0)$} & 8 & $P$ & 11 & 0.3095 & 0.3156 & 0.3133 \\
\hline & & $R$ & 11 & 0.2867 & 0.3092 & 0.2993 \\
\hline \multirow[t]{4}{*}{$(1,0,1) \leftarrow(0,1,1)^{\mathrm{b}}$} & 8 & $P_{1}$ & 10 & 0.1085 & 0.1398 & 0.1266 \\
\hline & & $P_{2}$ & 10 & 0.1403 & 0.1466 & 0.1438 \\
\hline & & $R_{1}$ & 10 & 0.0857 & 0.1358 & 0.1151 \\
\hline & & $R_{2}$ & 10 & 0.1144 & 0.1394 & 0.1279 \\
\hline$(1,1,0) \leftarrow(0,1,1)$ & 8 & $P$ & 11 & 0.0677 & 0.0980 & 0.0809 \\
\hline & & $Q$ & 11 & 0.0648 & 0.0752 & 0.0700 \\
\hline$(1,1,1) \leftarrow(0,1,0)$ & 8 & $Q$ & 11 & 0.2990 & 0.3056 & 0.3039 \\
\hline & & $R$ & 11 & 0.3077 & 0.3439 & 0.3256 \\
\hline$(1,1,0) \leftarrow(0,2,0)$ & 8 & $R$ & 8 & 0.0470 & 0.0498 & 0.0486 \\
\hline$(1,2,0) \leftarrow(0,1,0)$ & 8 & $P$ & 10 & 0.2776 & 0.3127 & 0.2969 \\
\hline$(1,2,0) \leftarrow(0,0,1)$ & 8 & $P$ & 9 & 0.0123 & 0.0313 & 0.0229 \\
\hline$(1,2,1) \leftarrow(0,0,2)^{b}$ & 10 & $P_{1}$ & 10 & 2.2235 & 3.7618 & 2.9684 \\
\hline & & $P_{2}$ & 10 & 2.2011 & 3.3771 & 2.7298 \\
\hline & & $Q_{1}$ & 8 & 2.3234 & 3.5923 & 2.9481 \\
\hline & & $Q_{2}$ & 9 & 2.2622 & 3.3879 & 2.7907 \\
\hline & & $R_{1}$ & 7 & 2.4657 & 3.6029 & 3.0344 \\
\hline & & $R_{2}$ & 7 & 2.3509 & 3.2274 & 2.7725 \\
\hline$(1,3,0) \leftarrow(0,0,0)$ & 16 & $P$ & 11 & 1.8857 & 2.6911 & 2.5708 \\
\hline & & $R$ & 8 & 2.5648 & 2.6808 & 2.6149 \\
\hline$(1,5,0) \leftarrow(0,1,0)$ & 17 & $P$ & 11 & 0.2277 & 0.3789 & 0.2952 \\
\hline & & $R$ & 11 & 0.2463 & 0.3685 & 0.2995 \\
\hline
\end{tabular}

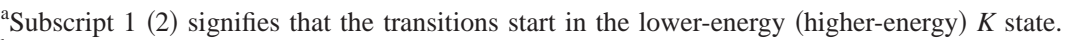

${ }^{\mathrm{b}}$ Subscript 1 (2) signifies that the transitions end in the lower energy (higher energy) $K$ state. 
TABLE VIII. Error statistics (in $\mathrm{cm}^{-1}$ ) for transitions in the CO overtone band; $N$ is the number of lines included from a given branch. Experimental results of McKellar (Ref. 15).

\begin{tabular}{cccccc}
\hline \hline Transition & Branch & $N$ & $\Delta_{\min }$ & $\Delta_{\max }$ & $\Delta_{\text {rms }}$ \\
\hline$(2,0,1) \leftarrow(0,0,0)$ & $P$ & 10 & 0.1355 & 0.1492 & 0.1421 \\
& $Q$ & 11 & 0.1282 & 0.1328 & 0.1311 \\
$(2,0,0) \leftarrow(0,0,1)$ & $R$ & 11 & 0.1203 & 0.1316 & 0.1259 \\
& $P$ & 11 & 0.0768 & 0.0837 & 0.0809 \\
& $Q$ & 11 & 0.0681 & 0.0754 & 0.0724 \\
& $R$ & 10 & 0.0550 & 0.0725 & 0.0640 \\
\hline \hline
\end{tabular}

\section{Rovibrational spectra}

Nearly 1000 rovibrational transitions of $\mathrm{Ar}-\mathrm{CO}$ have been experimentally observed and assigned, the majority of these embedded in the CO fundamental band around 2140 $\mathrm{cm}^{-1}$, and detailed comparison is obviously not practical. Instead, a comprehensive statistical comparison, encompassing a total of 720 lines, between available experimental rovibrational spectra and the theoretical results of the present work is given in this section. It must be stressed that the theoretical transition frequencies are calculated directly from the eigenvalues, not from the fitted energy levels of Sec. IV B. Furthermore, we shall refer to results as "experimental" even in cases where the transition frequency is not directly observed, e.g., due to coinciding $\mathrm{Ar}-\mathrm{CO}$ and $\mathrm{CO}$ absorptions, but rather calculated from experimentally deduced (i.e., fitted) energy levels.

The statistical quantities reported in Tables VI-VIII are the minimum and maximum absolute errors, $\Delta_{\min }$ and $\Delta_{\max }$, and the rms error, $\Delta_{\text {rms }}$, chosen in order to measure the error span and the typical error for a given rotational branch of each vibrational transition. In general, a small error span shows that the discrepancy is approximately independent of $J$, and therefore that the rotational structures of the vibrational levels involved are well described with the $\operatorname{CCSD}(\mathrm{T})$ IPESs.

Mostly, the errors are as expected from the analysis of the level origins and the spectroscopic constants of the $\nu_{\mathrm{vdW}}=0$ levels in the previous sections. Thus, the transitions solely involving the van der Waals ground state (i.e., $\nu_{\mathrm{vdW}}$ $=0 \leftarrow 0)$ are generally very well reproduced, although it is noted that those calculated in the $\mathrm{CO}$ overtone band are less accurate than in the fundamental band. This can, to some extent, be ascribed to the deficiencies of the $\operatorname{CCSD}(\mathrm{T})$ model in describing $\mathrm{CO}$ at bond distances outside the neighborhood of $r_{e}$.

Transitions involving excited van der Waals states, including those in which the high-lying $(1,5,0)$ level participates, display rms errors ranging from approximately 0.005 to $0.35 \mathrm{~cm}^{-1}$. The error spans indicate that the rotational structures of the $\nu_{\mathrm{vdW}}=1,2$, and 5 levels are somewhat less accurate but still comparable to that of the ground state. As anticipated, these comments do not apply to the $(1,2,1)$ $\leftarrow(0,0,2)$ and $(1,3,0) \leftarrow(0,0,0)$ bands. Not only are the rms errors atypical, but the error spans seemingly eradicate resemblance of the theoretical and experimental branch patterns of these bands.

One might argue that the discrepancy is readily resolved by simply interchanging the $(1,2,1)$ and $(1,3,0)$ levels, and then claim that the lines hitherto assigned to the former are really observations of the $(1,1,2)$ level. While some experimental features such as the asymmetry doubling of the transitions starting in the $(0,0,2)$ level would be preserved, others such as the lack of a $\mathrm{Q}$ branch of transitions from the $(0,0,0)$ level would not. Therefore, a theoretical intensity analysis is needed, taking into account the effect of temperature, in order to give a complete theoretical analysis of the spectrum in the "problematic" regions. To this end, we have initiated work involving ab initio calculation of the three-dimensional electric dipole surface of $\mathrm{Ar}-\mathrm{CO}$ to facilitate the calculation of rovibrational transition moments.

Disregarding the transitions to the $(1,2,1)$ and $(1,3,0)$ levels, the remaining 650 lines are reproduced with a total rms error of $0.13 \mathrm{~cm}^{-1}$. One should keep in mind that this result is somewhat biased by the over-representation of the van der Waals ground state in the statistics. Nevertheless, the total rms error warrants the assumption that the higher-lying van der Waals levels are predicted with an accuracy better than $1 \mathrm{~cm}^{-1}$.

\section{SUMMARY AND CONCLUDING REMARKS}

We have calculated the first three-dimensional $a b$ initio IPES for the Ar-CO van der Waals complex. Using a vibrationally adiabatic averaging procedure, the rovibrational energies and spectra have been calculated and compared to available experimental data. Energy levels are reported up to $50 \mathrm{~cm}^{-1}$ above the ground state. The statistical rms error of $0.13 \mathrm{~cm}^{-1}$ covering 650 experimentally observed transitions shows that the coupled cluster results may be used as a guide for future spectroscopic investigations of the $\mathrm{Ar}-\mathrm{CO}$ complex. However, we were not able to finally resolve the wellknown discrepancy between theory and experiment for the ordering of levels around $25 \mathrm{~cm}^{-1}$. For a complete simulation of the spectrum in this region, calculation of temperature-dependent spectral intensities is needed which, in turn, necessitates ab initio computation of the threedimensional electric dipole surface. Work along these lines is currently in progress.

Although the IPES is an example of state-of-the-art computational quantum chemistry, improvements may be obtained by explicitly allowing experimental information in the fitting procedure. For example, noting that the bending state is not as well described as with the aug-cc-pVTZ-33221 basis set, ${ }^{30}$ refitting the surface so as to (approximately) repro- 
duce the experimental frequency of this vibration, as done by Jansen to obtain the ECPF IPES, ${ }^{28}$ might enhance the quality.

\section{ACKNOWLEDGMENTS}

This work was supported by the EU-TMR network "Molecular Properties and Molecular Materials" (Contract No. HPRN-CT-2000-00013) and by the Spanish Comisión Interministerial de Ciencia y Tecnología (PB98-0609-C0401). H.K. acknowledges the Carlsberg Foundation. J.L.C. acknowledges an FPI grant from the Spanish Ministerio de Ciencia y Tecnología. The authors thank Dr. Christof Hättig for helpful comments.

${ }^{1}$ A. De Piante, E. J. Campbell, and S. J. Buelow, Rev. Sci. Instrum. 60, 858 (1989).

${ }^{2}$ A. R. W. McKellar, Y. P. Zeng, S. W. Sharpe, C. Wittig, and R. A. Beaudet, J. Mol. Spectrosc. 153, 475 (1992).

${ }^{3}$ T. Ogata, W. Jäger, I. Ozier, and M. C. L. Gerry, J. Chem. Phys. 98, 9399 (1993).

${ }^{4}$ M. Havenith, G. Hilpert, M. Petri, and W. Urban, Mol. Phys. 81, 1003 (1994).

${ }^{5}$ W. Jäger and M. C. L. Gerry, J. Chem. Phys. 102, 3587 (1995).

${ }^{6}$ S. König, G. Hilpert, and M. Havenith, Mol. Phys. 86, 1233 (1995).

${ }^{7}$ Y. Xu, S. Civiš, A. R. W. McKellar, S. König, M. Haverlag, G. Hilpert, and M. Havenith, Mol. Phys. 87, 1071 (1996).

${ }^{8}$ Y. Xu and A. R. W. McKellar, Mol. Phys. 88, 859 (1996).

${ }^{9}$ M. Hepp, W. Jäger, I. Pak, and G. Winnewisser, J. Mol. Spectrosc. 176, 58 (1996).

${ }^{10}$ S. König and M. Havenith, Mol. Phys. 91, 265 (1997).

${ }^{11}$ M. Hepp, R. Gendriesch, I. Pak, F. Lewen, and G. Winnewisser, J. Mol. Spectrosc. 183, 295 (1997).

${ }^{12}$ M. Hepp, R. Gendriesch, I. Pak, Y. A. Kuritsyn, F. Lewen, G. Winnewisser, M. Brookes, A. R. W. McKellar, J. K. G. Watson, and T. Amano, Mol. Phys. 92, 229 (1997).

${ }^{13}$ F. Lewen, R. Gendriesch, I. Pak, D. G. Paveliev, M. Hepp, P. Schneider, and G. Winnewisser, Rev. Sci. Instrum. 69, 32 (1998).

${ }^{14}$ R. Gendriesch, I. Pak, F. Lewen, L. Surin, D. A. Roth, and G. Winnewisser, J. Mol. Spectrosc. 196, 139 (1999).

${ }^{15}$ A. R. W. McKellar, Mol. Phys. 98, 111 (2000).

${ }^{16}$ I. Scheele, R. Lehnig, and M. Havenith, Mol. Phys. 99, 197 (2001).

${ }^{17}$ I. Scheele, R. Lehnig, and M. Havenith, Mol. Phys. 99, 205 (2001).

${ }^{18}$ D. G. Melnik, S. Gopalakrishnan, T. A. Miller, F. C. D. Lucia, and S. Belov, J. Chem. Phys. 114, 6100 (2001).

${ }^{19}$ L. A. Surin, B. S. Dumesh, F. Lewen, D. A. Roth, V. P. Kostromin, F. S. Rusin, G. Winnewisser, and I. Pak, Rev. Sci. Instrum. 72, 2535 (2001).

${ }^{20}$ G. A. Parker and R. T Pack, J. Chem. Phys. 69, 3268 (1978).

${ }^{21}$ K. Mirsky, Chem. Phys. 40, 445 (1980).

${ }^{22}$ J. Tennyson, S. Miller, and B. T. Sutcliffe, J. Chem. Soc., Faraday Trans. 2 84, 1295 (1988).

${ }^{23}$ C. A. Parish, J. D. Augspurger, and C. E. Dykstra, J. Phys. Chem. 96, 2069 (1992).

${ }^{24}$ G. Jansen, Chem. Phys. Lett. 223, 377 (1994).

${ }^{25}$ V. Castells, N. Halberstadt, S. K. Shin, R. A. Beaudet, and C. Wittig, J. Chem. Phys. 101, 1006 (1994).
${ }^{26}$ B. Kurkawska-Tarnawska, G. Chałasiński, and K. Olszewski, J. Chem. Phys. 101, 4964 (1994).

${ }^{27}$ S. Shin, S. K. Shin, and F.-M. Tao, J. Chem. Phys. 104, 183 (1996).

${ }^{28}$ G. Jansen, J. Chem. Phys. 105, 89 (1996).

${ }^{29}$ F. A. Gianturco, F. Paesani, M. F. Laranjeira, V. Vassilenko, and M. A. Cunha, J. Chem. Phys. 110, 7832 (1999).

${ }^{30}$ R. R. Toczyłowski and S. M. Cybulski, J. Chem. Phys. 112, 4604 (2000).

${ }^{31}$ F. A. Gianturco and F. Paesani, J. Chem. Phys. 115, 249 (2001).

${ }^{32}$ K. Raghavachari, G. W. Trucks, J. A. Pople, and M. Head-Gordon, Chem. Phys. Lett. 157, 479 (1989).

${ }^{33}$ C. Luo, R. Wehr, J. R. Drummond, A. D. May, F. Thibault, J. Boissoles, J. M. Launay, C. Boulet, J.-P. Bouanich, and J.-M. Hartmann, J. Chem. Phys. 115, 2198 (2001).

${ }^{34}$ B. T. Sutcliffe and J. Tennyson, Mol. Phys. 58, 1053 (1986).

${ }^{35}$ B. T. Sutcliffe and J. Tennyson, Int. J. Quantum Chem. 39, 183 (1991).

${ }^{36}$ D. M. Brink and G. R. Satchler, Angular Momentum, 2nd ed. (Clarendon, Oxford, 1968).

${ }^{37}$ J. Tennyson, S. Miller, and C. R. Le Sueur, Comput. Phys. Commun. 75, 339 (1993).

${ }^{38}$ B. Fernández, H. Koch, and J. Makarewicz, J. Chem. Phys. 110, 8525 (1999).

${ }^{39}$ H. Koch, B. Fernández, and J. Makarewicz, J. Chem. Phys. 111, 198 (1999).

${ }^{40}$ S. M. Cybulski and R. R. Toczyłowski, J. Chem. Phys. 111, 10520 (1999).

${ }^{41}$ R. Kobayashi, R. D. Amos, J. P. Reid, H. M. Quiney, and C. J. S. M. Simpson, Mol. Phys. 98, 1995 (2000).

${ }^{42}$ T. B. Pedersen, B. Fernández, H. Koch, and J. Makarewicz, J. Chem. Phys. 115, 8431 (2001).

${ }^{43}$ T. H. Dunning, J. Chem. Phys. 90, 1007 (1989).

${ }^{44}$ R. A. Kendall, T. H. Dunning, and R. J. Harrison, J. Chem. Phys. 96, 6796 (1992).

${ }^{45}$ D. E. Woon and T. H. Dunning, J. Chem. Phys. 98, 1358 (1993).

${ }^{46}$ D. E. Woon and T. H. Dunning, J. Chem. Phys. 100, 2975 (1994).

${ }^{47}$ S. F. Boys and F. Bernardi, Mol. Phys. 19, 553 (1970).

${ }^{48}$ T. Helgaker, H. J. Aa. Jensen, P. Jørgensen et al., DaLton, a Molecular Electronic Structure Program, Release 1.2, 2001, http://www.kjemi.uio.no/ software/dalton/dalton.html

${ }^{49}$ H. Koch, B. Fernández, and O. Christiansen, J. Chem. Phys. 108, 2784 (1998).

${ }^{50}$ K. L. Bak, J. Gauss, P. Jørgensen, J. Olsen, T. Helgaker, and J. F. Stanton, J. Chem. Phys. 114, 6548 (2001).

${ }^{51}$ T. G. A. Heijmen, R. Moszinsky, P. E. S. Wormer, and A. van der Avoird, J. Chem. Phys. 107, 9921 (1997).

${ }^{52}$ See EPAPS document No. E-JCPSA6-117-310231 for (a) the coordinates of the grid points and the values of the ab initio interaction energies, (b) a FORTRAN routine for generating the IPES, and (c) a FORTRAN routine for printing the rovibrational energies and spectra. This document may be retrieved via the EPAPS homepage (http://www.aip.org/pubservs/ epaps.html) or from ftp.aip.org in the directory /epaps/. See the EPAPS homepage for more information.

${ }^{53}$ R. Bukowski, J. Sadlej, B. Jeziorski, P. Jankowski, K. Szalewicz, S. A. Kucharski, H. L. Williams, and B. M. Rice, J. Chem. Phys. 110, 3785 (1999).

${ }^{54}$ P. Huxley and J. N. Murrel, J. Chem. Soc., Faraday Trans. 2 79, 323 (1983).

${ }^{55}$ K. Andersson, M. Barysz, A. Bernhardsson et al., MOLCAs, Version 5.2, Lund University, Sweden, 2001, http://www.teokem.lu.se/molcas/

${ }^{56}$ J. Tennyson and B. T. Sutcliffe, Mol. Phys. 58, 1067 (1986). 